\title{
MEAN EXIT TIME FOR SURFACE-MEDIATED DIFFUSION: SPECTRAL ANALYSIS AND ASYMPTOTIC BEHAVIOR
}

\author{
O. BÉNICHOU*, D. S. GREBENKOV ${ }^{\dagger}$, L. HILLAIRET ${ }^{\ddagger}$, L. PHUN ${ }^{\ddagger}$, R. VOITURIEZ*, AND \\ M. ZINSMEISTER ${ }^{\ddagger} \S$
}

\begin{abstract}
We consider a model of surface-mediated diffusion with alternating phases of pure bulk and surface diffusion. For this process, we compute the mean exit time from a disk through a hole on the circle. We develop a spectral approach to this escape problem in which the mean exit time is explicitly expressed through the eigenvalues of the related self-adjoint operator. This representation is particularly well suited to investigate the asymptotic behavior of the mean exit time in the limit of large desorption rate $\lambda$. For a point-like target, we show that the mean exit time diverges as $\sqrt{\lambda}$. For extended targets, we establish the asymptotic approach to a finite limit. In both cases, the mean exit time is shown to asymptotically increase as $\lambda$ tends to infinity. We also revise the optimality regime of surface-mediated diffusion. Although the presentation is limited to the unit disk, the spectral approach can be extended to other domains such as rectangles or spheres.
\end{abstract}

1. Introduction. Many transport and search processes exhibit intermittent character when different modes of motion are alternated. Typical examples are animals foraging (with phases of rapid relocation and slow exploration), facilitated search mechanism on DNA (with phases of pure bulk diffusion and chain sliding), vesicle transportation in living cells (with phases of active transport by motor proteins and passive diffusion in the cytoplasm), water transport in confining media (with phases of pure bulk diffusion and surface exploration) 3, 4. The intermittence is often expected to facilitate transport and search processes, e.g., by reducing the mean search time necessary to reach a target (food, specific DNA sequence, nucleus, or reaction zone in the above examples). In particular, the mean exit time from a bounded domain through an opening (a target) on the boundary has been actively studied during the last decade [9, 8. For pure bulk diffusion, Singer et al. derived the asymptotic behavior of the mean exit time in the narrow escape limit (when the size of the target is small) [21, 19, 20, 18, 10. Isaacson and Newby developed uniform in time asymptotic expansions in the target radius of the first passage time density for the diffusing molecule to find the target 7 . The escape problem for an intermittent process with phases of surface and pure bulk diffusion (the so-called surface-mediated diffusion) has been recently solved for rotation-invariant domains [1, 2]. The known eigenbases for the Laplace operators governing pure bulk and surface diffusions allowed one to express the mean exit time in a closed matrix form. Under well-defined conditions, the mean exit time was shown to be minimized at an optimal desorption rate that characterizes switching from surface to pure bulk diffusion. These results have been extended in various directions [16, 17, 5, 6]. An alternative master equation approach for discrete (on-lattice) surface-mediate diffusion (also called the bulk-mediated surface diffusion) has been proposed [11, 14, 12, 13.

In the present paper, we propose a rigorous spectral analysis of the above escape problem. We focus on surface-mediated diffusion in the unit disk and derive a spectral representation of the mean exit time. This representation is well suited to investigate

\footnotetext{
* Laboratoire de Physique Théorique de la Matière Condensée (UMR 7600), CNRS / UPMC, 4 Place Jussieu, 75255 Paris Cedex

†Laboratoire de Physique de la Matière Condensée (UMR 7643) CNRS - Ecole Polytechnique, 91128 Palaiseau, France

‡MAPMO (UMR 6628), Université d'Orléans, 45067 Orléans, France

$\S$ Corresponding author: zins@univ-orleans.fr
} 
the asymptotic behavior of the mean exit time in the limit of large desorption rate $\lambda$. For a point-like target, we show that the mean exit time diverges as $\sqrt{\lambda}$. For extended targets, we establish the asymptotic approach to a finite limit. In both cases, the mean exit time is shown to asymptotically increase as $\lambda$ tends to infinity. We also revise the optimality regime of surface-mediated diffusion. Although the presentation is limited to the unit disk, the spectral approach can be extended to other domains such as rectangles or spheres.

2. A self-adjoint operator formulation. We study the following model of surface-mediated diffusion in the unit disk $\mathbb{D}=\{z \in \mathbb{C}:|z|<1\}$ whose boundary $\partial \mathbb{D}$ includes an exit (or a target) of angular size $2 \epsilon$ (i.e., an arc of the unit circle between $\pi-\epsilon$ and $\pi+\epsilon$ ), with $0 \leq \epsilon \leq \pi$. A starting point $e^{i \theta}$ is taken on the unit circle. If the starting point is located on the target then the process is immediately stopped. Otherwise, the particle moves along the circle according to a Brownian motion with the diffusion coefficient $D_{1}$ for a duration of $\min \left\{\tau_{\lambda}, \tau\right\}$, where $\tau_{\lambda}$ is a random variable with exponential law of parameter $\lambda \geq 0$, and $\tau$ is the first hitting time of the target. If $\tau \leq \tau_{\lambda}$ then the process stops. If $\tau>\tau_{\lambda}$ then the particle is relocated at time $\tau_{\lambda}$ along the normal inside the disk at a distance $0<a \leq 1$ to start there a 2D Brownian motion with the diffusion coefficient $D_{2}$. This motion is stopped after hitting back the unit circle, and the same procedure is restarted from this last hitting point. We define $t_{1}(\theta)$ as being the expected time to reach the target. Similarly, for $0 \leq r<1$, we define $t_{2}\left(r e^{i \theta}\right)$ as being the expected time to reach the target starting from the point $r e^{i \theta}$ inside the unit disk.

It has been shown in [2] that these two functions satisfy the following system of equations:

$$
\left\{\begin{array}{l}
D_{1} t_{1}^{\prime \prime}(\theta)+\lambda\left[t_{2}\left((1-a) e^{i \theta}\right)-t_{1}(\theta)\right]=-1 \\
D_{2} \Delta t_{2}=-1 \\
t_{2}\left(e^{i \theta}\right)=t_{1}(\theta)(\theta \in[-\pi, \pi]) \\
t_{1}(\theta)=0 \text { if } \theta \in[-\pi,-\pi+\epsilon] \cup[\pi-\epsilon, \pi]
\end{array}\right.
$$

Let us notice that, by symmetry, $t_{1}(\theta)$ is an even function so it is sufficient to determine it on $[0, \pi]$.

The solution to Eq. (2.2) is the sum of the particular solution $\frac{1-r^{2}}{4 D_{2}}$ to the inhomogeneous (Poisson) equation $\Delta u=-\frac{1}{D_{2}},\left.u\right|_{\partial \mathbb{D}}=0$, and the solution to the Dirichlet problem $\Delta v=0,\left.v\right|_{\partial \mathbb{D}}=t_{1}$.

Since $t_{1}$ is even it may be represented as a cosine series

$$
t_{1}(\theta)=\sum_{n \geq 0} a_{n} \cos n \theta
$$

from which

$$
t_{2}\left(r e^{i \theta}\right)=\frac{1-r^{2}}{4 D_{2}}+\sum_{n \geq 0} a_{n} r^{n} \cos n \theta
$$

Eq. (2.1) then becomes

$$
t_{1}^{\prime \prime}(\theta)=-\frac{1}{D_{1}}\left(1+\lambda \frac{1-(1-a)^{2}}{4 D_{2}}\right)+\frac{\lambda}{D_{1}} U\left(t_{1}\right)
$$


where $U$ is the operator on $L^{2}([0, \pi])$ defined by

$$
U\left(\sum_{n \geq 0} x_{n} \cos n \theta\right)=\sum_{n \geq 1} x_{n}\left(1-(1-a)^{n}\right) \cos n \theta
$$

This operator can also be written as $U=V^{2}$, where

$$
V\left(\sum_{n \geq 0} x_{n} \cos n \theta\right)=\sum_{n \geq 1} x_{n} \sqrt{1-(1-a)^{n}} \cos n \theta
$$

Next we introduce the Sturm Liouville operator $T$ defined on $L^{2}([0, \pi-\epsilon])$ as $T f=u$, where

$$
\left\{\begin{array}{l}
u^{\prime \prime}=f \\
u^{\prime}(0)=u(\pi-\epsilon)=0 .
\end{array}\right.
$$

The operator $T$ is negative self-adjoint. Finally, we define $\tilde{T}=-E T R$ as an operator on $L^{2}([0, \pi])$, where $R: L^{2}([0, \pi]) \longrightarrow L^{2}([0, \pi-\epsilon])$ is the natural restriction, and $E: L^{2}([0, \pi-\epsilon]) \longrightarrow L^{2}([0, \pi])$ is the natural extension by 0 . The operator $\tilde{T}$ can be written explicitly as

$$
\tilde{T}(f)= \begin{cases}\int_{\theta}^{\pi-\epsilon} d \theta_{1} \int_{0}^{\theta_{1}} d \theta_{2} f\left(\theta_{2}\right), & 0 \leq \theta<\pi-\epsilon \\ 0, & \pi-\epsilon \leq \theta \leq \pi\end{cases}
$$

One can easily check that the eigenbasis of this operator is

$$
\nu_{n}=\frac{(1-\epsilon / \pi)^{2}}{(n+1 / 2)^{2}}, \quad u_{n}= \begin{cases}\sqrt{\frac{2}{\pi-\epsilon}} \cos \left(\frac{(n+1 / 2) \theta}{1-\epsilon / \pi}\right), & 0 \leq \theta \leq \pi-\epsilon, \\ 0, & \pi-\epsilon \leq \theta \leq \pi .\end{cases}
$$

These eigenvectors form an orthogonal basis of $L^{2}[0, \pi-\epsilon]$ by Sturm-Liouville theory.

Let us check that $\tilde{T}$ is a self-adjoint non-negative operator on $L^{2}([0, \pi])$. Let $f$, $g$ be in $L^{2}([0, \pi])$ :

$$
\begin{aligned}
-\langle\tilde{T} f, \bar{g}\rangle & =\langle E T R f, \bar{g}\rangle=\int_{0}^{\pi-\epsilon} T(R f) \bar{g}=\int_{0}^{\pi-\epsilon} T(R f) \overline{R g} \\
& =\int_{0}^{\pi-\epsilon} R f \overline{T(R g)}=\int_{0}^{\pi} f \overline{\operatorname{ETR}(g)}=-\langle f, \overline{\tilde{T} g}\rangle,
\end{aligned}
$$

which proves the claim, since the operator $E T R$ is negative.

The operator $\tilde{T}$ allows us to translate Eq. (2.5) into

$$
t_{1}=\frac{1}{D_{1}}\left(1+\lambda \frac{1-(1-a)^{2}}{4 D_{2}}\right) \tilde{T}(1)-\frac{\lambda}{D_{1}} \tilde{T} U\left(t_{1}\right) .
$$

We next apply the operator $V$ to both sides of Eq. 2.11) to get, writing $s_{1}=V\left(t_{1}\right)$,

$$
s_{1}=\frac{1}{D_{1}}\left(1+\lambda \frac{1-(1-a)^{2}}{4 D_{2}}\right) V \tilde{T}(1)-\frac{\lambda}{D_{1}} V \tilde{T} V\left(s_{1}\right)
$$


which can be solved in $s_{1}$ as

$$
s_{1}=\frac{1}{D_{1}}\left(1+\lambda \frac{1-(1-a)^{2}}{4 D_{2}}\right)\left(I+\frac{\lambda}{D_{1}} V \tilde{T} V\right)^{-1}(\psi),
$$

where $\psi=V \tilde{T}(1)$. This is an exact solution of the original problem for a fixed starting point. We emphasize that the operators $V$ and $\tilde{T}$, as well as the function $\psi=V \tilde{T}(1)$, are given explicitly. At first thought, this representation looks similar to the mean exit time found in [2] (see also [16, 17]). Although both derivations are conceptually similar, the major advantage of the present approach is the use of the self-adjoint operator $V \tilde{T} V$. This feature allows one to invert the operator $\left(I+\frac{\lambda}{D_{1}} V \tilde{T} V\right)$ in Eq. (2.14) and to express the mean exit time in a spectral form (see below).

The case of a randomly distributed starting point on the circle with uniform law is of particular interest. This is equivalent to averaging the mean exit time over the starting points that we denote as

$$
\left\langle t_{1}\right\rangle=\frac{1}{\pi} \int_{0}^{\pi} d \theta t_{1}(\theta)=\frac{1}{\pi}\left\langle t_{1}, 1\right\rangle .
$$

Using Eqs. (2.11) and (2.12), we can write

$$
\begin{aligned}
\pi\left\langle t_{1}\right\rangle & =\frac{1}{D_{1}}\left(1+\lambda \frac{1-(1-a)^{2}}{4 D_{2}}\right)\langle\tilde{T}(1), 1\rangle-\frac{\lambda}{D_{1}}\left\langle\tilde{T} V\left(s_{1}\right), 1\right\rangle \\
& =\frac{1}{D_{1}}\left(1+\lambda \frac{1-(1-a)^{2}}{4 D_{2}}\right)\langle\tilde{T}(1), 1\rangle-\frac{\lambda}{D_{1}}\left\langle s_{1}, \psi\right\rangle,
\end{aligned}
$$

from which it follows that the knowledge of $s_{1}$ allows to compute $\left\langle t_{1}\right\rangle$ :

$$
\left\langle t_{1}\right\rangle=\frac{1}{\pi D_{1}}\left(1+\lambda \frac{1-(1-a)^{2}}{4 D_{2}}\right)\left(\langle\tilde{T}(1), 1\rangle-\frac{\lambda}{D_{1}}\left\langle\left(I+\frac{\lambda}{D_{1}} V \tilde{T} V\right)^{-1} \psi, \psi\right\rangle\right) .
$$

By spectral theorem there exists an orthonormal basis of $L^{2}([0, \pi])$ which diagonalizes the self-adjoint operator $V \tilde{T} V$. More precisely, $L^{2}([0, \pi])$ is the orthogonal direct sum of $\operatorname{ker}(V \tilde{T} V)$ and $\operatorname{Im}(V \tilde{T} V)$ and we obtain this orthonormal basis by completing any orthonormal basis of $\operatorname{ker}(V \tilde{T} V)$ with the basis formed by the normalized eigenvectors associated with positive eigenvalues.

To identify these two spaces let us notice first that $\operatorname{ker} V$ is the one dimensional space of constant functions. Thus $\operatorname{ker}(V \tilde{T} V)$ is the space of functions $f \in L^{2}([0, \pi])$ such that $\tilde{T}(V f)$ is constant. But since $V f=\tilde{T}(V f)^{\prime \prime}$ on $[0, \pi-\epsilon], V f \equiv 0$ on $[0, \pi-\epsilon]$. So $f \in \operatorname{ker}(V \tilde{T} V) \Rightarrow \operatorname{supp}(V f) \subset[\pi-\epsilon, \pi]$, and this implication is easily seen to be an equivalence. With a slight abuse of language, we write $\operatorname{ker}(V \tilde{T} V)=$ $V^{-1}\left(L^{2}(\pi-\epsilon, \pi)\right)$. It follows that $\operatorname{Im}(V \tilde{T} V)=V\left(L^{2}([0, \pi-\epsilon])\right)$.

We call $\left(e_{n}\right)_{n \geq 0}$ the orthonormal basis of $\operatorname{Im}(V \tilde{T} V)$ such that $V \tilde{T} V e_{n}=\lambda_{n} e_{n}$ and $\lambda_{n} \downarrow 0$ as $n \rightarrow \infty$.

When $\epsilon=0$, the eigenbasis $e_{n}$ is simply formed by cosine functions, and the analysis is straightforward (see below). When $\epsilon>0$, we first observe that $\psi=$ $V \tilde{T}(1)=V \tilde{T}\left(\varphi_{0}\right)$ where

$$
\varphi_{0}= \begin{cases}1 & \text { on }[0, \pi-\epsilon[ \\ -\frac{\pi-\epsilon}{\epsilon} & \text { on }[\pi-\epsilon, \pi]\end{cases}
$$


so that $\int_{0}^{\pi} \varphi_{0}=0$, and thus there exists $\psi_{0} \in L^{2}([0, \pi])$ such that $\varphi_{0}=V \psi_{0}$. It follows that $\psi \in \operatorname{Im}(V \tilde{T} V)$ and we can write

$$
\psi=\sum_{n \geq 1} \psi_{n} e_{n}
$$

with coefficients $\psi_{n}$ forming a sequence in $\ell^{2}$. Using this representation, Eq. (2.12) is formally solved as

$$
s_{1}=\frac{1}{D_{1}}\left(1+\lambda \frac{1-(1-a)^{2}}{4 D_{2}}\right) \sum_{n \geq 1} \frac{\psi_{n}}{1+\frac{\lambda}{D_{1}} \lambda_{n}} e_{n} .
$$

Plugging this expression into Eq. (2.14) we obtain

$$
\left\langle t_{1}\right\rangle=\frac{1}{\pi D_{1}}\left(1+\lambda \frac{1-(1-a)^{2}}{4 D_{2}}\right)\left[\langle\tilde{T}(1), 1\rangle-\frac{\lambda}{D_{1}} \sum_{n \geq 0} \frac{\psi_{n}^{2}}{1+\frac{\lambda}{D_{1}} \lambda_{n}}\right] .
$$

This spectral representation is particularly well suited for the asymptotic analysis of the mean exit time.

2.1. Point-like target $(\epsilon=0)$. We first consider the case of $\epsilon=0$. Although such a target is not accessible for $2 \mathrm{D}$ pure bulk diffusion, it can still be reached through 1D surface diffusion. In this case, one easily gets

$$
\left\{\begin{array}{l}
\tilde{T}(\cos n \theta)=-T(\cos n \theta)=\frac{\cos n \theta-(-1)^{n}}{n^{2}} \quad(n \geq 1), \\
\tilde{T}(1)=-T(1)=\frac{\pi^{2}-\theta^{2}}{2},
\end{array}\right.
$$

so that

$$
\left\{\begin{array}{l}
V \tilde{T} V(\cos n \theta)=\frac{1-(1-a)^{n}}{n^{2}} \cos n \theta \quad(n \geq 1) \\
V \tilde{T} V(1)=0
\end{array}\right.
$$

One concludes that

$$
\lambda_{n}=\left\{\begin{array}{ll}
\frac{1-(1-a)^{n}}{n^{2}} & (n \geq 1), \\
0 & (n=0),
\end{array} \quad e_{n}= \begin{cases}\sqrt{2 / \pi} \cos n \theta & (n \geq 1), \\
\sqrt{1 / \pi} & (n=0) .\end{cases}\right.
$$

For $n \geq 1$, we have

$$
\begin{aligned}
\psi_{n} & =\left\langle\psi, e_{n}\right\rangle=\left\langle V \tilde{T}(1), e_{n}\right\rangle=\sqrt{2 / \pi}\langle\tilde{T}(1), V(\cos n \theta)\rangle \\
& =\sqrt{2 / \pi} \sqrt{1-(1-a)^{n}}\left\langle\frac{\pi^{2}-\theta^{2}}{2}, \cos n \theta\right\rangle=\sqrt{2 \pi} \sqrt{1-(1-a)^{n}} \frac{(-1)^{n+1}}{n^{2}}
\end{aligned}
$$

while $\langle\psi, 1\rangle=\langle V \tilde{T}(1), 1\rangle=\langle\tilde{T}(1), V 1\rangle=0$. Substituting this expression into Eq. (2.15), we get

$$
\begin{aligned}
\left\langle t_{1}\right\rangle_{\epsilon=0} & =\frac{1}{\pi D_{1}}\left(1+\lambda \frac{1-(1-a)^{2}}{4 D_{2}}\right)\left(\langle\tilde{T}(1), 1\rangle-\sum_{n \geq 1} \frac{2 \pi \frac{\lambda}{D_{1}}\left(1-(1-a)^{n}\right)}{n^{2}\left(n^{2}+\frac{\lambda}{D_{1}}\left(1-(1-a)^{n}\right)\right)}\right) \\
& =\frac{1}{\pi D_{1}}\left(1+\lambda \frac{1-(1-a)^{2}}{4 D_{2}}\right)\left(\langle\tilde{T}(1), 1\rangle-2 \pi \sum_{n \geq 1} \frac{1}{n^{2}}\right. \\
& \left.+2 \pi \sum_{n \geq 1} \frac{1}{n^{2}+\frac{\lambda}{D_{1}}\left(1-(1-a)^{n}\right)}\right) .
\end{aligned}
$$


From Eq. (2.16), we get

$$
\langle\tilde{T}(1), 1\rangle_{\epsilon=0}=\int_{0}^{\pi} d \theta \frac{\pi^{2}-\theta^{2}}{2}=\frac{1}{3} \pi^{3} .
$$

We also know the value of the Riemann zeta function

$$
\zeta(2)=\sum_{n \geq 1} \frac{1}{n^{2}}=\frac{\pi^{2}}{6} .
$$

Plugging Eqs. (2.21) and (2.22) into Eq. (2.20) yields

$$
\left\langle t_{1}\right\rangle_{\epsilon=0}=\frac{2}{D_{1}}\left(1+\lambda \frac{1-(1-a)^{2}}{4 D_{2}}\right) \sum_{n \geq 1} \frac{1}{n^{2}+\frac{\lambda}{D_{1}}\left(1-(1-a)^{n}\right)} .
$$

We retrieved the exact representation of the mean exit time for point-like target that was first derived in [1].

For $0<a<1$, in the following inequalities

$$
\sum_{n \geq 1} \frac{1}{n^{2}+\frac{\lambda}{D_{1}}} \leq \sum_{n \geq 1} \frac{1}{n^{2}+\left(1-(1-a)^{n}\right) \frac{\lambda}{D_{1}}} \leq \sum_{n \geq 1} \frac{1}{n^{2}+a \frac{\lambda}{D_{1}}},
$$

Since $f(x)=\frac{1}{x^{2}+\frac{a \lambda}{D_{1}}}$ is decreasing, by applying the property that $\int_{n}^{n+1} f(x) d x \leq$ $f(n) \leq \int_{n-1}^{n} f(x) d x$, one can replace sums by integrals to get

$$
\sqrt{\frac{D_{1}}{\lambda}} \frac{\pi}{2} F\left(D_{1} / \lambda\right) \leq \sum_{n \geq 1} \frac{1}{n^{2}+\left(1-(1-a)^{n}\right) \frac{\lambda}{D_{1}}} \leq \sqrt{\frac{D_{1}}{a \lambda}} \frac{\pi}{2},
$$

where $F(x)=1-\frac{2}{\pi} \arctan (\sqrt{x})$. One obtains therefore

$$
\frac{\pi F\left(D_{1} / \lambda\right)}{\sqrt{D_{1} \lambda}}\left(1+\lambda \frac{1-(1-a)^{2}}{4 D_{2}}\right) \leq\left\langle t_{1}\right\rangle_{\epsilon=0} \leq \frac{\pi}{\sqrt{a D_{1} \lambda}}\left(1+\lambda \frac{1-(1-a)^{2}}{4 D_{2}}\right) .
$$

In the limit of large $\lambda, F(x)$ can be approximated by 1, i.e.

$$
\frac{1-(1-a)^{2}}{4 D_{2} \sqrt{D_{1}}} \pi \sqrt{\lambda}+\frac{\pi}{\sqrt{D_{1} \lambda}} \lesssim\left\langle t_{1}\right\rangle_{\epsilon=0} \leq \frac{1-(1-a)^{2}}{4 D_{2} \sqrt{D_{1} a}} \pi \sqrt{\lambda}+\frac{\pi}{\sqrt{D_{1} a \lambda}} .
$$

Therefore, we conclude that $\left\langle t_{1}\right\rangle_{\epsilon=0}$ asymptotically increases to infinity, as illustrated on Fig. 2.1 $\mathrm{a}$. As a result, if $\left.\frac{\partial\left\langle t_{1}\right\rangle_{\epsilon=0}}{\partial \lambda}\right|_{\lambda=0}<0$, then $\left\langle t_{1}\right\rangle_{\epsilon=0}$ has a minimum. We compute

$$
\begin{aligned}
& \left.\frac{\partial\left\langle t_{1}\right\rangle_{\epsilon=0}}{\partial \lambda}\right|_{\lambda=0}=\frac{2}{D_{1}}\left(\frac{1-(1-a)^{2}}{4 D_{2}} \sum_{n \geq 1} \frac{1}{n^{2}}-\frac{1}{D_{1}} \sum_{n \geq 1} \frac{1-(1-a)^{n}}{n^{4}}\right)<0 \\
& \Leftrightarrow \frac{1-(1-a)^{2}}{4 D_{2}} \frac{\pi^{2}}{6}<\frac{1}{D_{1}} \sum_{n \geq 1} \frac{1-(1-a)^{n}}{n^{4}} \\
& \Leftrightarrow D_{2}>D_{2, \text { crit }}=D_{1} \frac{\pi^{2}\left(1-(1-a)^{2}\right)}{24 \sum_{n \geq 1} \frac{1-(1-a)^{n}}{n^{4}}}
\end{aligned}
$$


We retrieved the optimality condition first reported in [1. The relation (2.26) determines the critical value of the pure bulk diffusion coefficient $D_{2, \text { crit }}$, which for small a can be approximated as

$$
\lim _{a \rightarrow 0} D_{2, \mathrm{crit}}=D_{1} \frac{\pi^{2}}{12 \zeta(3)} \approx 0.68 D_{1} .
$$

If $D_{2}>D_{2, \text { crit }}$, then $\left\langle t_{1}\right\rangle_{\epsilon=0}$ has a minimum.

FIG. 2.1. Mean exit time $\left\langle t_{1}\right\rangle$ as a function of $\lambda$ for $\epsilon=0$ (a) and $\epsilon=0.01$ (b), with $a=0.01$

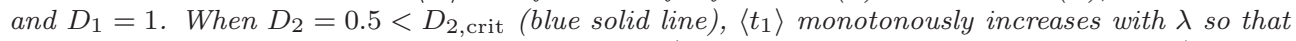
the smallest mean exit time corresponds to $\lambda=0$ (surface diffusion without intermittence). When $D_{2}=2>D_{2, \text { crit }}$ (red dashed line), $\left\langle t_{1}\right\rangle$ starts first to decrease with $\lambda$, passes through a minimum and monotonously increases to infinity. For point-like target (a), thin solid lines indicate the leading term of the lower bound $\frac{1-(1-a)^{2}}{4 D_{2} \sqrt{D_{1}}} \pi \sqrt{\lambda}$ from Eq. 2.25). Note that the upper bound, which is larger by $a^{-1 / 2}$, strongly overestimates the mean exit time. Finally, correction terms of the order $\lambda^{-1 / 2}$ are negligible for large $\lambda$. For extended target (b), horizontal lines indicate the limiting values of the mean exit time as $\lambda \rightarrow \infty$. Symbols present the diagonal approximation (4.3).
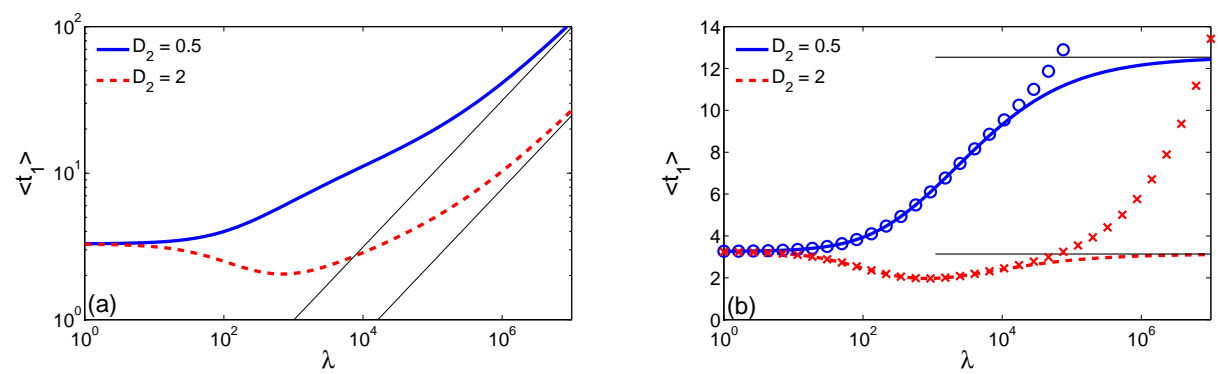

2.2. Extended target $(\epsilon>0)$. In sharp contrast to a point-like target, the mean exit time $\left\langle t_{1}\right\rangle$ to an extended target $(\epsilon>0)$ converges in a finite limit as $\lambda \rightarrow \infty$. As a consequence, the quantity $\langle\tilde{T}(1), 1\rangle-\frac{\lambda}{D_{1}} \sum_{n \geq 0} \frac{\psi_{n}^{2}}{1+\frac{\lambda}{D_{1}} \lambda_{n}}$ must converge to 0 as $\lambda \rightarrow \infty$. Given that

$$
\lim _{\lambda \rightarrow \infty} \sum_{n \geq 1} \frac{\lambda \psi_{n}^{2}}{1+\frac{\lambda}{D_{1}} \lambda_{n}}=D_{1} \sum_{n \geq 1} \frac{\psi_{n}^{2}}{\lambda_{n}}
$$

we deduce

$$
\langle\tilde{T}(1), 1\rangle=\sum_{n \geq 1} \frac{\psi_{n}^{2}}{\lambda_{n}}
$$

and this series converges. Setting $\lambda=0$ into Eq. (2.15), the above expression can be identified to the mean exit time for surface diffusion phase:

$$
\left\langle t_{1}\right\rangle_{\lambda=0}=\frac{1}{\pi D_{1}} \sum_{n \geq 1} \frac{\psi_{n}^{2}}{\lambda_{n}}=\frac{1}{\pi D_{1}}\langle\tilde{T}(1), 1\rangle=\frac{(\pi-\epsilon)^{3}}{3 \pi D_{1}}
$$

because $\tilde{T}(1)=\frac{(\pi-\epsilon)^{2}-\theta^{2}}{2}$ for $0 \leq \theta<\pi-\epsilon$, and 0 otherwise.

Plugging Eq. (2.28) into Eq. (2.15), we obtain

$$
\left\langle t_{1}\right\rangle=\frac{1}{\pi}\left(\frac{1}{\lambda}+\frac{1-(1-a)^{2}}{4 D_{2}}\right) \sum_{n \geq 1} \frac{\psi_{n}^{2}}{\lambda_{n}\left(\frac{D_{1}}{\lambda}+\lambda_{n}\right)} .
$$


This formula generalizes Eq. (2.23) to extended targets.

Similarly, we have

$$
\lim _{\lambda \rightarrow \infty} \sum_{n \geq 1} \frac{\psi_{n}^{2}}{\lambda_{n}\left(\frac{D_{1}}{\lambda}+\lambda_{n}\right)}=\sum_{n \geq 1} \frac{\psi_{n}^{2}}{\lambda_{n}^{2}}
$$

so that

$$
\mathcal{T}=\lim _{\lambda \rightarrow \infty}\left\langle t_{1}\right\rangle=\frac{1-(1-a)^{2}}{4 \pi D_{2}} \sum_{n \geq 1} \frac{\psi_{n}^{2}}{\lambda_{n}^{2}}
$$

i.e., we got a spectral representation for the time $\mathcal{T}$ corresponding to pure bulk diffusion. Note also that the series $S=\sum_{n \geq 1} \frac{\psi_{n}^{2}}{\lambda_{n}^{2}}$ converges.

We may now state the main theorem of this work:

THEOREM 2.1. The function $\lambda \mapsto\left\langle t_{1}\right\rangle$ is eventually increasing as $\lambda \rightarrow+\infty$. Moreover, if

$$
\left.\frac{d\left\langle t_{1}\right\rangle}{d \lambda}\right|_{\lambda=0}<0
$$

then the function $\left\langle t_{1}\right\rangle$ passes through a minimum.

Proof.

LEMma 2.2 .

$$
\sum_{n \geq 1} \frac{\psi_{n}^{2}}{\lambda_{n}^{3}}=+\infty
$$

The proof of this lemma will be given in the next section. We assume it is true for the rest of the proof of the main theorem.

We rewrite Eq. (2.30) as follows

$$
\left\langle t_{1}\right\rangle=\mathcal{T}+\frac{S}{\pi \lambda}-\frac{D_{1}}{\lambda} \frac{1-(1-a)^{2}}{4 \pi D_{2}} \sum_{n \geq 1} \frac{\psi_{n}^{2}}{\lambda_{n}^{2}\left(\lambda_{n}+\frac{D_{1}}{\lambda}\right)}-\frac{D_{1}}{\pi \lambda^{2}} \sum_{n \geq 1} \frac{\psi_{n}^{2}}{\lambda^{2}\left(\lambda_{n}+\frac{D_{1}}{\lambda}\right)} .
$$

Since

$$
\lim _{\lambda \rightarrow \infty} \sum_{n \geq 1} \frac{\psi_{n}^{2}}{\lambda_{n}^{2}\left(\lambda_{n}+\frac{D_{1}}{\lambda}\right)}=\sum_{n \geq 1} \frac{\psi_{n}^{2}}{\lambda_{n}^{3}}=+\infty
$$

we see that

$$
\left\langle t_{1}\right\rangle=\mathcal{T}-\frac{D_{1}}{\lambda} \frac{1-(1-a)^{2}}{4 \pi D_{2}} \sum_{n \geq 1} \frac{\psi_{n}^{2}}{\lambda_{n}^{2}\left(\lambda_{n}+\frac{D_{1}}{\lambda}\right)}+O\left(\lambda^{-1}\right),
$$

from which the principle part of $\left\langle t_{1}\right\rangle$ is indeed non-decreasing as $\lambda \rightarrow \infty$.

To complete the proof, we differentiate Eq. (2.32) (the exchange of derivative and sum is easily established):

$$
\pi \frac{d\left\langle t_{1}\right\rangle}{d \lambda}=-\frac{S}{\lambda^{2}}+\frac{1}{\lambda^{2}} \frac{D_{1}\left(1-(1-a)^{2}\right)}{4 D_{2}} \sum_{n \geq 1} \frac{\psi_{n}^{2}}{\lambda_{n}\left(\lambda_{n}+\frac{D_{1}}{\lambda}\right)^{2}}+\frac{D_{1}}{\lambda^{3}} \sum_{n \geq 0} \frac{\psi_{n}^{2}\left(2 \lambda_{n}+D_{1} / \lambda\right)}{\lambda_{n}^{2}\left(\lambda_{n}+D_{1} / \lambda\right)^{2}}
$$


which becomes positive at large $\lambda$ because

$$
\lim _{\lambda \rightarrow \infty} \sum_{n \geq 1} \frac{\psi_{n}^{2}}{\lambda_{n}} \frac{1}{\left(\lambda_{n}+\frac{D_{1}}{\lambda}\right)^{2}}=\sum_{n \geq 1} \frac{\psi_{n}^{2}}{\lambda_{n}^{3}}=+\infty .
$$

Returning to formula (2.15), we have

$$
\begin{aligned}
\pi \frac{d\left\langle t_{1}\right\rangle}{d \lambda}= & \frac{1-(1-a)^{2}}{4 D_{1} D_{2}}\left[\langle\tilde{T}(1), 1\rangle-\frac{\lambda}{D_{1}} \sum_{n \geq 1} \frac{\psi_{n}^{2}}{1+\frac{\lambda \lambda_{n}}{D_{1}}}\right] \\
& +\frac{1}{D_{1}}\left(1+\frac{\lambda\left(1-(1-a)^{2}\right)}{4 D_{2}}\right)\left[-\frac{1}{D_{1}} \sum_{n \geq 1} \frac{\psi_{n}^{2}}{1+\frac{\lambda \lambda_{n}}{D_{1}}}+\frac{\lambda}{D_{1}} \sum_{n \geq 1} \frac{\psi_{n}^{2} \frac{\lambda_{n}}{D_{1}}}{\left(1+\frac{\lambda \lambda_{n}}{D_{1}}\right)^{2}}\right] .
\end{aligned}
$$

In particular, one gets

$$
\begin{aligned}
\left.\pi \frac{d\left\langle t_{1}\right\rangle}{d \lambda}\right|_{\lambda=0} & =\frac{1-(1-a)^{2}}{4 D_{1} D_{2}}\langle\tilde{T}(1), 1\rangle+\frac{1}{D_{1}^{2}}\left[-\sum_{n \geq 1} \psi_{n}^{2}\right] \\
& =\frac{1-(1-a)^{2}}{4 D_{1} D_{2}}\langle\tilde{T}(1), 1\rangle-\frac{1}{D_{1}^{2}}\|V \tilde{T}(1)\|^{2}
\end{aligned}
$$

which becomes negative when

$$
\begin{gathered}
\|V \tilde{T}(1)\|^{2}>\frac{D_{1}}{4 D_{2}}\left(1-(1-a)^{2}\right)\langle\tilde{T}(1), 1\rangle \\
\Leftrightarrow\left\|V \tilde{T} V\left(\psi_{0}\right)\right\|^{2}>\frac{D_{1}}{4 D_{2}}\left(1-(1-a)^{2}\right)\left\langle V \tilde{T} V\left(\psi_{0}\right), \psi_{0}\right\rangle .
\end{gathered}
$$

Note that

$$
\begin{aligned}
\psi & =V \tilde{T}(1)=V\left(\frac{(\pi-\epsilon)^{2}-\theta^{2}}{2}\right)=V\left(\frac{2}{\pi} \sum_{n \geq 1}^{\infty} \cos n \theta\left\langle\frac{(\pi-\epsilon)^{2}-\theta^{2}}{2}, \cos n \theta\right\rangle\right) \\
& =\frac{2}{\pi} \sum_{n \geq 1}^{\infty} \cos n \theta \sqrt{1-(1-a)^{n}}\left\langle\frac{(\pi-\epsilon)^{2}-\theta^{2}}{2}, \cos n \theta\right\rangle \\
& =\frac{2}{\pi} \sum_{n \geq 1}^{\infty} \cos n \theta \sqrt{1-(1-a)^{n}}(-1)^{n-1} \frac{(\pi-\epsilon) \cos n \epsilon+\frac{\sin n \epsilon}{n}}{n^{2}} .
\end{aligned}
$$

REMARK 2.1. The inequality (2.33) determines the critical value for the pure bulk diffusion coefficient $D_{2, \text { crit }}$ above which pure bulk excursions are beneficial. The existence of the optimal value $\lambda$ (that minimizes the function $\left\langle t_{1}\right\rangle$ ) depends on this ratio. If $D_{2}>D_{2, \text { crit }}$, with

$$
\begin{aligned}
D_{2, \text { crit }} & =D_{1} \frac{\left(1-(1-a)^{2}\right)\langle\tilde{T}(1), 1\rangle}{4\|V \tilde{T}(1)\|^{2}} \\
& =D_{1} \frac{\pi(\pi-\epsilon)^{3}\left(1-(1-a)^{2}\right)}{24}\left(\sum_{n \geq 1} \frac{1-(1-a)^{n}}{n^{4}}\left[(\pi-\epsilon) \cos n \epsilon+\frac{\sin n \epsilon}{n}\right]^{2}\right)^{-1}
\end{aligned}
$$


then $\left\langle t_{1}\right\rangle$ starts first to decrease with $\lambda$, passes through a minimum then monotonously increases. We retrieved the optimality condition first reported in [1].

Proof. [Proof of the main lemma] First we define $\tilde{e}_{n}=\frac{1}{\lambda_{n}} \tilde{T} V e_{n}$ so that $V \tilde{e}_{n}=e_{n}$.

Let $u$ be such that $\psi=V \tilde{T} V(u) ; u$ must be of the form $\psi_{0}+u^{\perp}$, where $u^{\perp} \in$ $\operatorname{ker}(V \tilde{T} V)$. Let

$$
u_{n}=\left\langle u, e_{n}\right\rangle=\frac{1}{\lambda_{n}}\left\langle u, V \tilde{T} V e_{n}\right\rangle=\frac{1}{\lambda_{n}}\left\langle V \tilde{T} V u, e_{n}\right\rangle=\frac{\psi_{n}}{\lambda_{n}} .
$$

REMARK 2.2. This computation gives another proof that $\sum_{n \geq 1} \frac{\psi_{n}^{2}}{\lambda_{n}^{2}}<\infty$ and thus also that $\sum_{n \geq 1} \frac{\psi_{n}^{2}}{\lambda_{n}}<\infty$.

Now, on $[0, \pi-\epsilon],-\lambda_{n} \tilde{e}_{n}^{\prime \prime}=V e_{n}$ because $\tilde{e}_{n}=\frac{1}{\lambda_{n}} \tilde{T} V e_{n}$. Let $m, n$ be integers, and we establish:

$$
\lambda_{n}\left\langle\tilde{e}_{n}^{\prime}, \tilde{e}_{m}^{\prime}\right\rangle=-\lambda_{n}\left\langle\tilde{e}_{n}^{\prime \prime}, \tilde{e}_{m}\right\rangle=\left\langle V e_{n}, \tilde{e}_{m}\right\rangle=\left\langle e_{n}, V \tilde{e}_{m}\right\rangle=\left\langle e_{n}, e_{m}\right\rangle=\delta_{m, n} .
$$

Setting $\epsilon_{n}=\sqrt{\lambda_{n}} \tilde{e}_{n}$, we get that $\left(\epsilon_{n}^{\prime}\right)$ is an orthonormal system of $L^{2}([0, \pi])$.

Next assume that $\sum_{n \geq 1} \frac{\psi_{n}^{2}}{\lambda_{n}^{3}}<\infty$ : then the above computation shows that $\sum_{n \geq 1} \frac{\psi_{n}}{\lambda_{n}} \tilde{e}_{n}$ is a function of the Sobolev space $H^{1}([0, \pi])$.

Before we continue let us observe that the operator $I-V$ is regularizing: $\forall f \in$ $L^{2}([0, \pi]), V f=f+g$ where $g \in C^{\infty}([0, \pi])$.

This implies that

$$
V\left(\sum_{n \geq 1} \frac{\psi_{n}}{\lambda_{n}} \tilde{e}_{n}\right)=\sum_{n \geq 1} \frac{\psi_{n}}{\lambda_{n}} \tilde{e}_{n}+g, \quad g \in C^{\infty} .
$$

On the other hand

$$
V\left(\sum_{n \geq 1} \frac{\psi_{n}}{\lambda_{n}} \tilde{e}_{n}\right)=\sum_{n \geq 1} \frac{\psi_{n}}{\lambda_{n}} e_{n}
$$

and thus $u_{0}=\sum_{n \geq 1} \frac{\psi_{n}}{\lambda_{n}} e_{n} \in H^{1}$.

But $u_{0}$ minimizes $\|v\|_{2}^{2}$ on the set of $v$ such that $\int_{0}^{\pi}(V v-1)^{2}=0, \int_{\pi-\epsilon}^{\pi} V v=$ $-(\pi-\epsilon)$.

By the theory of constrained extrema, $v$ must be of the form $\lambda V 1_{[\pi-\epsilon, \pi[}=$ $\lambda 1_{[\pi-\epsilon, \pi[}+g$ with $g \in C^{\infty}$. But such a function cannot be in $H^{1}$.

We have thus proven the main lemma.

3. Asymptotic behavior. Here we analyze the asymptotic behavior of $\left\langle t_{1}\right\rangle$ as $\lambda \rightarrow \infty$.

For point-like target $(\epsilon=0)$, Eqs. (2.18) and (2.19) imply for small $a$ the existence of two distinct asymptotic behaviors:

$$
\begin{aligned}
& \lambda_{n} \simeq \frac{a}{n}, \quad \psi_{n}^{2} \simeq \frac{2 \pi a}{n^{3}} \quad(n \ll 1 / a), \\
& \lambda_{n} \simeq \frac{1}{n^{2}}, \quad \psi_{n}^{2} \simeq \frac{2 \pi}{n^{4}} \quad(n \gg 1 / a) .
\end{aligned}
$$


FIG. 3.1. Eignvalues $\lambda_{n}$ of the operator $V \tilde{T} V$ for (a) $a=0.001$ and three values $\epsilon: 0.01$ (circles), 0.1 (crosses), and 1 (triangles); and (b) for $\epsilon=0.01$ and three values of a: 0.001 (circles), 0.01 (crosses), and 0.1 (triangles). Solid lines show the asymptotic relations $a / n$ and $1 / n^{2}$, while vertical dotted lines indicate the separation $1 /$ a between these asymptotic regimes. The coefficient $A_{\epsilon}$ in front of $n^{-2}$ relation is close to 1 for all small targets, except for $\epsilon=1$, see Eq. (3.4).
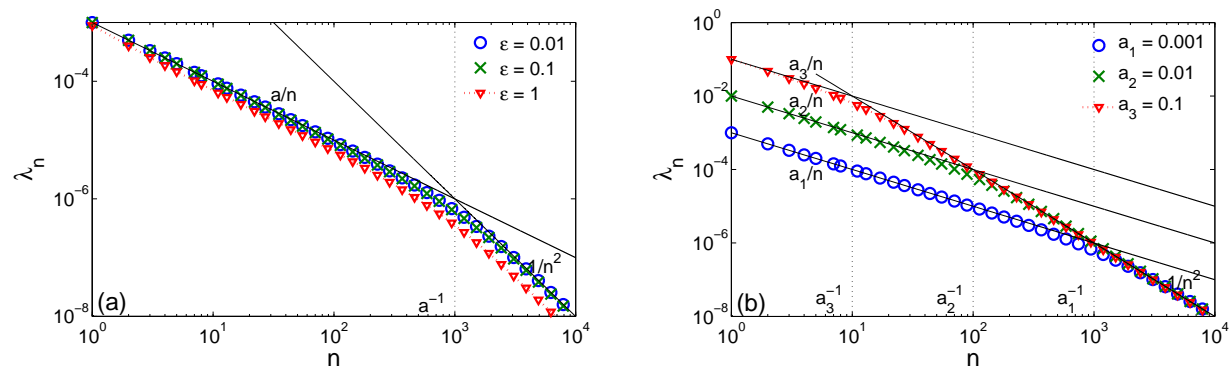

In this section, we analyze the case of extended targets $(\epsilon>0)$ by numerical computations of the eigenvalues $\lambda_{n}$ and the spectral weights $\psi_{n}^{2}$ (see Appendix B for computational details). As for the point-like target, Figure 3.1 allows one to distinguish two regimes for $\lambda_{n}$, for small and large $n$ :

$$
\lambda_{n} \simeq \begin{cases}\tilde{A}_{a, \epsilon} n^{-1} & (n \ll 1 / a), \\ A_{\epsilon} n^{-2} & (n \gg 1 / a),\end{cases}
$$

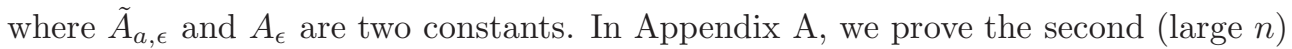
asymptotic relation and show that the constant $A_{\epsilon}$ does not depend on $a$. In turn, the transition between two asymptotic regimes is determined by $1 / a$ (and is independent of $\epsilon$ ). Note also that $A_{\epsilon} \rightarrow 1$ as $\epsilon \rightarrow 0$ according to Eq. (3.2). One can see that these asymptotic relations accurately approximate the eigenvalues $\lambda_{n}$. The behavior of $A_{\epsilon}$ is shown on Fig. 3.3 . As expected, it does not depend on $a$. These numerical results suggest the following conjectural expression:

$$
A_{\epsilon}=(1-\epsilon / \pi)^{2}
$$

which accurately reproduces $A_{\epsilon}$ on the whole range of $\epsilon$ from 0 to $\pi$. According to Eq. (3.1), the coefficient $\tilde{A}_{a, \epsilon}$ is equal to $a$ when $\epsilon=0$. We plot therefore $\tilde{A}_{a, \epsilon} / a$ on Fig. 3.3 b, where this ratio approaches 1 as $\epsilon \rightarrow 0$, and 0 as $\epsilon \rightarrow \pi$. Moreover, this ratio weakly depends on $a$ (curves for $a=0.001$ and $a=0.1$ almost coincide).

Figure 3.2 shows that the asymptotic behavior of the spectral weights $\psi_{n}^{2}$ is more complicated. One can distinguish three asymptotic regimes:

$$
\psi_{n}^{2} \simeq \begin{cases}\tilde{B}_{a, \epsilon} n^{-3} & (n \ll \min \{1 / a, 1 / \epsilon\}), \\ \tilde{B}_{a, \epsilon}^{\prime} n^{-4} & (\min \{1 / a, 1 / \epsilon\} \ll n \ll \max \{1 / a, 1 / \epsilon\}), \\ B_{a, \epsilon} n^{-6} & (n \gg \max \{1 / a, 1 / \epsilon\}),\end{cases}
$$

In order to observe all three regimes, one needs $1 \ll \min \{1 / a, 1 / \epsilon\} \ll \max \{1 / a, 1 / \epsilon\}$, i.e., either $a \ll \epsilon \ll 1$, or $\epsilon \ll a \ll 1$. For instance, if $a$ or $\epsilon$ is not small enough, the first regime with $n^{-3}$ may not be well established (Fig. 3.2 a, b). If $a \sim \epsilon$, the intermediate regime disappears, as illustrated on Fig. 3.2. Finally, when $\epsilon \rightarrow 0$, $\max \{1 / a, 1 / \epsilon\} \rightarrow \infty$, the third regime disappears, and one retrieves two regimes for point-like targets. 
FIG. 3.2. Spectral weights $\psi_{n}^{2}$ (shown by red solid line) for (a) $a=0.001$ and $\epsilon=0.1$; (b) $a=0.1$ and $\epsilon=0.001$; and (c) $a=\epsilon=0.01$. In the first two plots, three asymptotic regimes can be distinguished according to Eq. [3.5), while the intermediate regime disappears in the last plot.
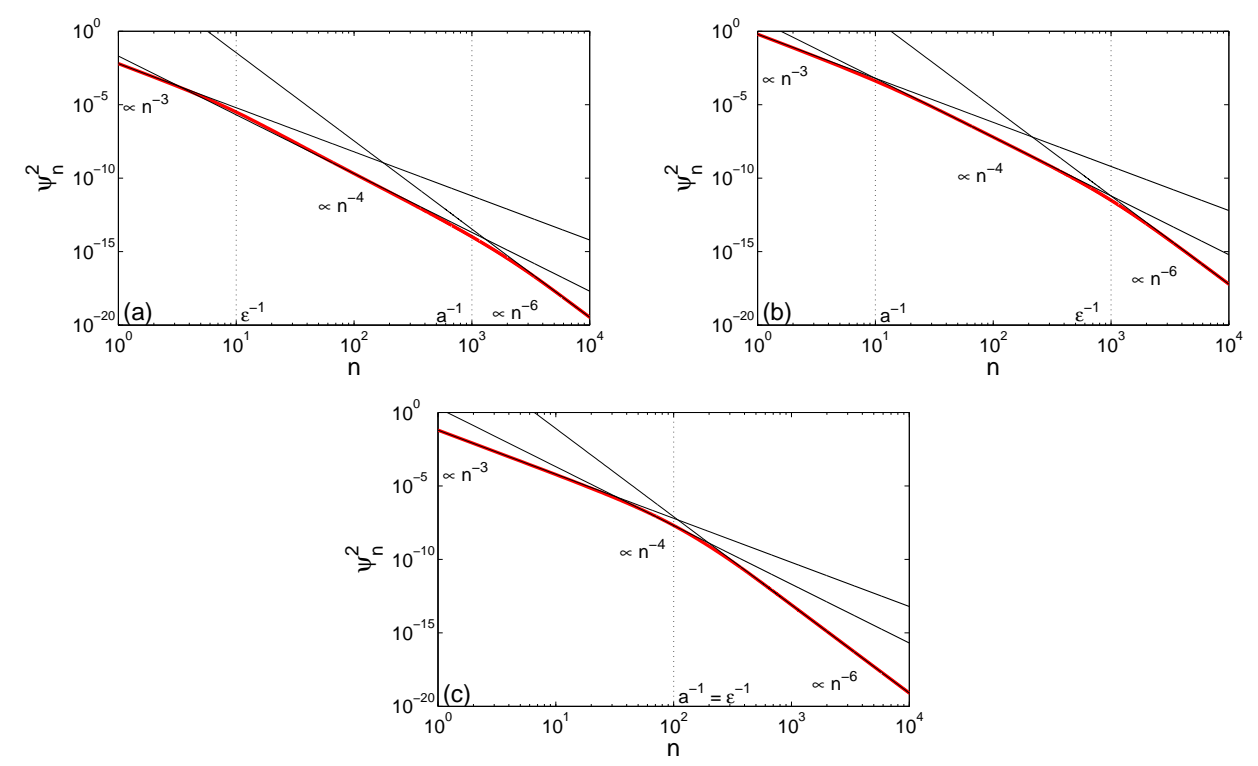

The behavior of the coefficients $B_{a, \epsilon}$ and $\tilde{B}_{a, \epsilon}$ is shown on Fig. 3.3., d. As expected from Eq. (3.1), $\tilde{B}_{a, \epsilon} /(2 \pi a)$ approaches 1 as $\epsilon \rightarrow 0$ (point-like target). Moreover, such normalized coefficient weakly depends on $a$ (at least for small $a$ ). The behavior of $B_{a, \epsilon}$ is more complicated. Given that $\mathcal{T}$ should converge to the mean exit time for pure bulk diffusion, $\left\langle t_{1}\right\rangle_{b}$, as $a \rightarrow 0$, one gets

$$
\sum_{n \geq 1} \frac{\psi_{n}^{2}}{\lambda_{n}^{2}} \simeq \frac{2 \pi D_{2}\left\langle t_{1}\right\rangle_{b}}{a}
$$

Since $\lambda_{n} \simeq A_{\epsilon} n^{-2}$ is independent of $a$, one concludes that $B_{a, \epsilon} \sim 1 / a$ as $a \rightarrow 0$. For this reason, we plot $a B_{a, \epsilon}$ on Fig. 3.3k. For large $\epsilon$, two curves for $a=0.001$ and $a=0.1$ do coincide, as expected. However, strong deviations emerge at small $\epsilon$. In fact, one needs to consider much smaller $a$ to get coinciding curves over the whole considered range of $\epsilon$. We conclude that the reflection distance $a$ plays an important role, especially for small targets.

Although the above asymptotic regimes for $\lambda_{n}$ and $\psi_{n}^{2}$ remain conjectural, we will investigate their consequences for the asymptotic behavior of the mean exit time $\left\langle t_{1}\right\rangle$. Using the asymptotic relations for large $n$, we get

$$
\begin{aligned}
\sum_{n \geq 1} \frac{\psi_{n}^{2}}{\lambda_{n}^{2}\left(\lambda_{n}+\frac{D_{1}}{\lambda}\right)} & \sim \int_{1}^{\infty} \frac{B_{a, \epsilon} x^{-6}}{A_{\epsilon}^{2} x^{-4}\left(A_{\epsilon} x^{-2}+\frac{D_{1}}{\lambda}\right)} d x \\
& =\frac{B_{a, \epsilon}}{A_{\epsilon}^{3}} \int_{1}^{\infty} \frac{d x}{1+x^{2} \frac{D_{1}}{\lambda A_{\epsilon}}} \sim \frac{B_{a, \epsilon}}{A_{\epsilon}^{5 / 2}} \frac{\pi}{2} \sqrt{\frac{\lambda}{D_{1}}} .
\end{aligned}
$$


FIg. 3.3. Coefficients $A_{\epsilon}, \tilde{A}_{a, \epsilon} / a, a B_{a, \epsilon}$, and $\tilde{B}_{a, \epsilon} / a$ from Eqs. 3.3), 3.5 versus $\epsilon$. Two curves for $a=0.001$ and $a=0.1$ coincide that illustrates the independence of $A_{\epsilon}$ and $\tilde{A}_{\epsilon} / a$ of $a$.
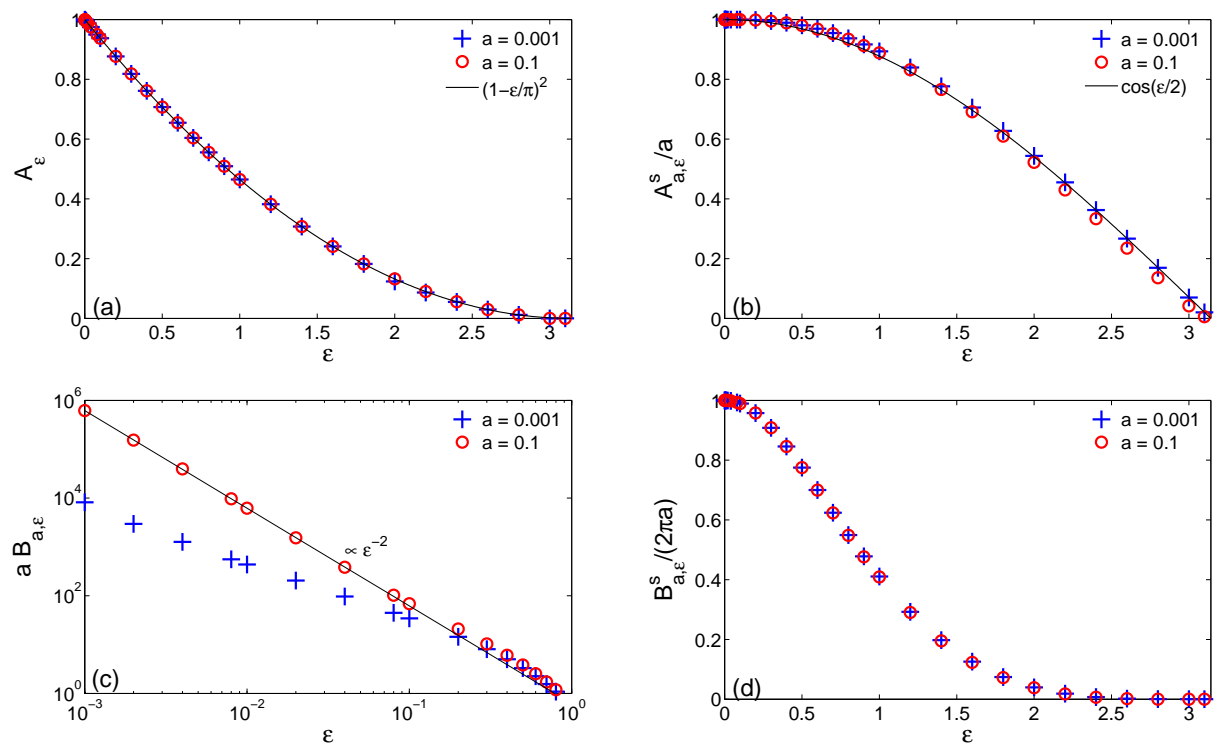

Consequently, we get

$$
\left\langle t_{1}\right\rangle=\mathcal{T}-\frac{C_{1}}{\sqrt{\lambda}}+O\left(\frac{1}{\lambda}\right)
$$

where

$$
C_{1}=C_{a, \epsilon} \frac{\sqrt{D_{1}}}{D_{2}}, \quad C_{a, \epsilon}=\left(1-(1-a)^{2}\right) \frac{B_{a, \epsilon}}{8 A_{\epsilon}^{5 / 2}}
$$

For an accurate numerical computation of $\left\langle t_{1}\right\rangle$, we consider the behavior of partial sums $f(N)=\sum_{n=1}^{N} \frac{\psi_{n}^{2}}{\lambda_{n}\left(\frac{D_{1}}{\lambda}+\lambda_{n}\right)}$ (note that $\left\langle t_{1}\right\rangle$ is obtained in the limit $N \rightarrow \infty$ according to Eq. (2.30) ). We checked that $f(N) \sim f(\infty)+\frac{c}{N}$ for large $N$ (for a fixed $\lambda)$. In practice, we used the fourth order polynomial fit of $f(N)$ versus $1 / N$ for $N$ from 1000 to 20000 to extrapolate the value $f(\infty)$.

Figure 3.4 shows the mean exit time $\left\langle t_{1}\right\rangle$ as a function of $\lambda$ for a small target $(\epsilon=0.01)$ and two values of $a$ : 0.01 and 0.001 . In both cases, the mean exit time passes through a minimum at some intermediate desorption rate $\lambda_{c}$ and then approaches the maximum as $\lambda \rightarrow \infty$. One can clearly see that the optimal value $\lambda_{c}$, as well as the height of the maximum at $\lambda \rightarrow \infty$, depend on $a$. Although both considered values $a=0.001$ and $a=0.01$ are small, the limiting mean exit time $\mathcal{T}$ changes significantly. The asymptotic relation (3.7) (shown by thin solid lines) accurately captures the limiting behavior. 
FIG. 3.4. The mean exit time $\left\langle t_{1}\right\rangle$ as a function of $\lambda$ for $\epsilon=0.01, D_{1}=D_{2}=1$, and two values of a: 0.01 (solid line) and 0.001 (dashed line). Two horizontal lines indicate the mean exit times for surface diffusion $\left\langle t_{1}\right\rangle_{\lambda=0} \approx 3.2586$ and for pure bulk diffusion $\left\langle t_{1}\right\rangle_{\lambda=\infty} \approx 5.2929$ from Eqs. [2.29), C.5. Thin lines show the asymptotic behavior (3.7) where $\mathcal{T}$ and $C_{1}$ are computed from Eqs. 2.31, 3.8.

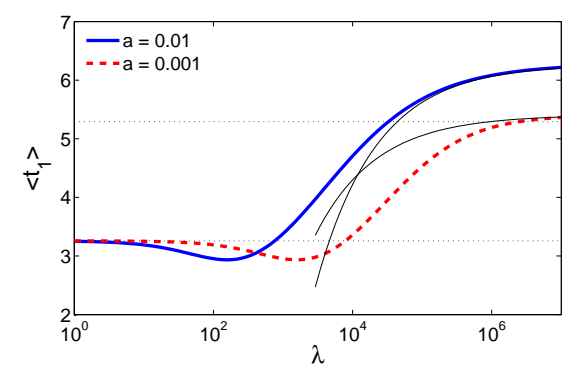

When $\epsilon \rightarrow 0$, we use Eqs. 3.3.3) and (3.5) to get

$$
\begin{aligned}
\mathcal{T} & =\frac{1-(1-a)^{2}}{4 \pi D_{2}} \sum_{n \geq 1} \frac{\psi_{n}^{2}}{\lambda_{n}^{2}}=\frac{1-(1-a)^{2}}{4 \pi D_{2}}\left(\sum_{n=1}^{1 / \epsilon} \frac{\tilde{B}_{a, \epsilon} n^{-3}}{\tilde{A}_{a, \epsilon}^{2} n^{-2}}+O(\epsilon)\right) \\
& =\frac{1-(1-a)^{2}}{4 \pi D_{2}}\left(\frac{\tilde{B}_{a, \epsilon}}{\tilde{A}_{a, \epsilon}^{2}} \ln \frac{1}{\epsilon}+O(\epsilon)\right) .
\end{aligned}
$$

This logarithmic divergence is similar to the result from Ref. 21, 19, 20, which describes the mean exit time for non-intermittent pure bulk diffusion $(2 \mathrm{D}$ Brownian motion) in the narrow escape limit $(\epsilon \rightarrow 0)$. This case formally corresponds to the double limit $\lambda \rightarrow \infty$ and $a \rightarrow 0$. Interestingly, the double limit can be taken separately: as $\lambda \rightarrow \infty$, the limiting value $\mathcal{T}$ exists for any finite $a$. In this way, we extended the classical escape problem for pure bulk diffusion by including reflections at a finite distance $a$. In Appendix C, we provide the exact formula for the mean exit time for pure bulk diffusion $\left\langle t_{1}\right\rangle_{b}$. In the narrow escape limit $\epsilon \rightarrow 0$, one gets $\left\langle t_{1}\right\rangle_{b} \approx \frac{\ln (2 / \epsilon)}{D_{2}}(1+O(\epsilon))$.

Figure 3.5 shows the dependence of the limiting mean exit time $\mathcal{T}$ on $a$ and $\epsilon$. For fixed target size $\epsilon, \mathcal{T}$ approaches the mean exit time for pure bulk diffusion $\left\langle t_{1}\right\rangle_{b}$ as $a \rightarrow 0$ (Fig. [3.5 ). Note that a simple formula for $\mathcal{T}$ in the special case $a=1$ is derived in Appendix $\mathrm{D}$. For fixed reflection distance $a, \mathcal{T}$ exhibits the logarithmic dependence on $\epsilon$ for $\epsilon \gtrsim a$, in agreement with Eq. (3.9). In the opposite case $\epsilon \lesssim a$, the reflection distance $a$ alters this behavior.

4. Diagonal approximation. In [3, an explicit diagonal approximation to the mean exit time has been proposed and checked numerically to be accurate. In our notations, this approximation consists in removing all nondiagonal elements of the matrix VTV from Eq. (B.6) that represents the operator $V \tilde{T} V$ in the cosine basis. Under this approximation, one gets

$$
\lambda_{n} \approx \frac{1-(1-a)^{n}}{n^{2}} \frac{\pi-\epsilon+\frac{\sin 2 n \epsilon}{2 n}}{\pi}, \quad e_{n} \approx \sqrt{\frac{2}{\pi}} \cos (n \theta),
$$

from which

$$
\psi_{n}^{2} \approx \frac{2\left(1-(1-a)^{n}\right)}{\pi n^{4}}\left((\pi-\epsilon) \cos n \epsilon+\frac{\sin n \epsilon}{n}\right)^{2} .
$$


FIG. 3.5. (a) The limiting mean exit time $\mathcal{T}$ as a function of a for $\epsilon=0.01$ (circles) and $\epsilon=0.1$ (crosses), $D_{2}=1$. Two horizontal lines indicate the mean exit time for pure bulk diffusion $\left\langle t_{1}\right\rangle_{\lambda=\infty}$ : 5.2929 and 2.9949, respectively. As expected, $\mathcal{T}$ increases with a because every reflection from the boundary by distance a requires additional time to return (given that the target is located at the boundary). In the limit $a=1, \mathcal{T}$ becomes 78.2898 for $\epsilon=0.01$ and 7.6040 for $\epsilon=0.1$ according to Eq. (D.1). (b) $\mathcal{T}$ as a function of $\epsilon$ for $a=0.001$ (pluses) and $a=0.01$ (circles). Solid line shows the mean exit time for pure bulk diffusion from Eq. C.5. As expected, the limiting time diverges logarithmically with $\epsilon$ when $\epsilon \gtrsim a$. In the opposite case $\epsilon \lesssim a$, the reflection distance a alters this behavior.
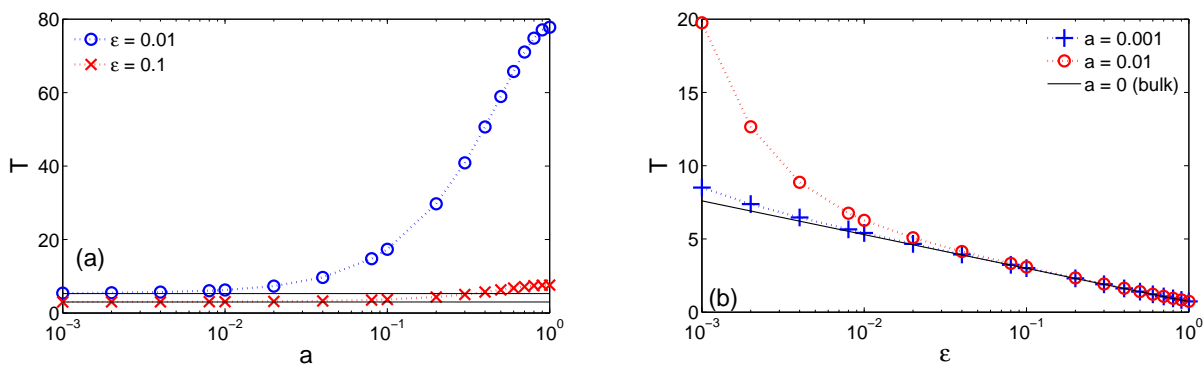

Substituting these approximations into Eq. (2.15), we retrieve the diagonal approximation from [3]:

$$
\begin{aligned}
\left\langle t_{1}\right\rangle & \approx \frac{1}{\pi D_{1}}\left(1+\lambda \frac{1-(1-a)^{2}}{4 D_{2}}\right)\left[\frac{(\pi-\epsilon)^{3}}{3}\right. \\
& \left.-\frac{2 \lambda}{\pi D_{1}} \sum_{n \geq 1} \frac{1-(1-a)^{n}}{n^{2}} \frac{\left((\pi-\epsilon) \cos n \epsilon+\frac{\sin n \epsilon}{n}\right)^{2}}{n^{2}+\frac{\lambda}{\pi D_{1}}\left(1-(1-a)^{n}\right)\left(\pi-\epsilon+\frac{\sin 2 n \epsilon}{2 n}\right)}\right] .
\end{aligned}
$$

The diagonal approximation has been shown numerically to be very accurate for moderate $\lambda$ and arbitrary $\epsilon$ (see [3]). Although the matrix VTV has indeed the dominant diagonal, the remarkable quality of this approximation remained puzzling. At the same time, the approximation becomes inappropriate in the limit of large $\lambda$. In order to get a finite limit of the mean exit time for $\epsilon>0$ as $\lambda \rightarrow \infty$, the constant term $(\pi-\epsilon)^{3} / 3$ has to be compensated. In other words, writing Eq. (4.3) as

$$
\begin{aligned}
\left\langle t_{1}\right\rangle & \approx \frac{1}{\pi D_{1}}\left(1+\lambda \frac{1-(1-a)^{2}}{4 D_{2}}\right)\left\{\left[\frac{(\pi-\epsilon)^{3}}{3}-2 \sum_{n \geq 1} \frac{\left((\pi-\epsilon) \cos n \epsilon+\frac{\sin n \epsilon}{n}\right)^{2}}{\pi-\epsilon+\frac{\sin 2 n \epsilon}{2 n}} \frac{1}{n^{2}}\right]\right. \\
& \left.+2 \sum_{n \geq 1} \frac{\left((\pi-\epsilon) \cos n \epsilon+\frac{\sin n \epsilon}{n}\right)^{2}}{\pi-\epsilon+\frac{\sin 2 n \epsilon}{2 n}} \frac{1}{n^{2}+\frac{\lambda}{\pi D_{1}}\left(1-(1-a)^{n}\right)\left(\pi-\epsilon+\frac{\sin 2 n \epsilon}{2 n}\right)}\right\},
\end{aligned}
$$

the constant term in square brackets should be canceled. This is true only for $\epsilon=0$ and $\epsilon=\pi$. As a consequence, the diagonal approximation (4.3) (or (4.4) implies the divergence of the mean exit time as $\lambda \rightarrow \infty$, in contrast to the expected convergence to a finite limit for $\epsilon>0$.

This discrepancy can be interpreted in terms of the asymptotic behavior of the spectral weights $\psi_{n}^{2}$. In fact, the approximate relation (4.2) suggests $\psi_{n}^{2} \propto n^{-4}$, in contrast to the observed $n^{-6}$ decay. In other words, the diagonal approximation operates over an intermediate asymptotic regime, while the ultimate $n^{-6}$ asymptotics 
is missed. This observation explains why the diagonal approximation accurately captures the behavior of the mean exit time $\left\langle t_{1}\right\rangle$ over small and moderate $\lambda$ but fails in the limit $\lambda \rightarrow \infty$ (see Fig. 2.1b).

Conclusion. In summary, we presented a spectral approach for computing the mean exit time $\left\langle t_{1}\right\rangle$ from the unit disk by surface-mediated diffusion with two alternating phases of bulk and surface motion. The derived spectral representation is particularly suitable for the asymptotic analysis of the mean exit time in the limit of large desorption rates $\lambda$. For a point-like target $(\epsilon=0)$, we proved the asymptotic increase $\left\langle t_{1}\right\rangle \propto \sqrt{\lambda}$ and provided lower and upper bounds. For extended targets $(\epsilon>0)$, we investigated the asymptotic approach of the mean exit time to a finite limit and proved an increase of $\left\langle t_{1}\right\rangle$ at large $\lambda$ (the first correction term being $\propto \lambda^{-1 / 2}$ ). We revealed different asymptotic behaviors of the governing eigenvalues $\lambda_{n}$ and spectral weights $\psi_{n}^{2}$ depending on the target size $\epsilon$ and the reflection distance $a$. Finally, we discussed the optimality of surface-mediated diffusion and potential limitations of the diagonal approximation for the mean exit time.

\section{Appendices.}

A. Asymptotic behavior of the eigenvalues of the operator $V \tilde{T} V$. THEOREM A.1. Let $\lambda_{n}$ be the eigenvalues of the operator $V \tilde{T} V$, where the operators $\tilde{T}$ and $V$ are defined in Eqs. (2.7) and (2.6). We have

$$
\lambda_{n} \sim A_{\epsilon} n^{-2}
$$

where $A_{\epsilon}$ depends only on $\epsilon$.

Proof. In order to prove this statement, we first investigate the following problem:

Let $A$ and $B$ are two compact, positive, self-adjoint operators. We assume that the eigenvalues of the operator $A$ are ordered in a decreasing sequence: $\lambda_{1}(A) \geq$ $\lambda_{2}(A) \geq \ldots \geq \lambda_{n}(A) \geq \ldots \geq 0$. We recall the variational principle as following

THEOREM A.2.

$$
\left.\lambda_{n}(A)=\max _{F} \min _{O \neq x \in A} \frac{\langle A x, x\rangle}{\langle x, x\rangle} ; \text { (where } F \text { is a subspaces of } L^{2}[0, \pi] ; \operatorname{dim} F=n\right) .
$$

The max is taken over $F$, the subspace associated with the first $n$ eigenvectors of $A$. We state two following lemmas which will be needed to prove the proposition A.1

Lemma A.3. We make the assumption that $\lambda_{n}(A), \lambda_{n}(A+B)$ are the $n^{\text {th }}$ eigenvalues of the operators $A$ and $A+B$. Then, we have

$$
\lambda_{n}(A)-\|B\| \leq \lambda_{n}(A+B) \leq \lambda_{n}(A)+\|B\|,
$$

where $\|$.$\| define the norm of an operator in L^{2}[0, \pi]$ space.

Proof. Let $F$ be the subspace of $L^{2}[0, \pi]$ associated with the first $n$ eigenvectors of $A$. For all $x \in F$, we have

$$
\langle(A+B) x, x\rangle=\langle A x, x\rangle+\langle B x, x\rangle .
$$

According to the variational principle, we have

$$
\langle A x, x\rangle \geq \min _{x \in A} \frac{\langle A x, x\rangle}{\langle x, x\rangle}\|x\|^{2}=\lambda_{n}(A)\|x\|^{2} .
$$


Besides, we have

$$
|\langle B x, x\rangle| \leq\|B\|\|x\|^{2} .
$$

It follows from Eqs. A.4 and (A.5) that

$$
\langle(A+B) x, x\rangle \geq \lambda_{n}(A)\|x\|^{2}-\|B\|\|x\|^{2} .
$$

This gives

$$
\min _{0 \neq x \in F} \frac{\langle(A+B) x, x\rangle}{\|x\|^{2}} \geq \lambda_{n}(A)-\|B\| .
$$

Again, according to the variational principle, we thus get

$$
\lambda_{n}(A+B) \geq \lambda_{n}(A)-\|B\| .
$$

In the same manner, if we take $F$ be associated to the first $n$ eigenvectors of $A+B$, we can get

$$
\lambda_{n}(A+B) \leq \lambda_{n}(A)+\|B\|,
$$

and the lemma $\mathrm{A.3}$ follows. $\mathrm{\square}$

Lemma A.4. With the notations used in lemma A.3, if $\operatorname{rank}(B)<\infty$, then

$$
\lambda_{n+\operatorname{rank}(B)}(A) \leq \lambda_{n}(A+B) \leq \lambda_{n-\operatorname{rank}(B)}(A) .
$$

Proof. We call $F$ the subspace of $L^{2}[0, \pi]$ associated with the first $n-\operatorname{rank}(B)$ eigenvectors of $A$.

By the variational principle, we have

$$
\forall x \in F \cap \operatorname{ker}(B),\|x\|=1:\langle(A+B) x, x\rangle=\langle A x, x\rangle \geq \lambda_{n+\operatorname{rank}(B)}(A) .
$$

Consequently,

$$
\min _{x \in F \cap \operatorname{ker}(B) ;\|x\|=1}\langle(A+B) x, x\rangle \geq \lambda_{n+\operatorname{rank}(B)}(A) .
$$

Since

$$
\operatorname{dim}(F \cap \operatorname{ker}(B))=\operatorname{dim} F-\operatorname{dim} B(F) \geq n,
$$

we have

$$
\lambda_{n}(A+B) \geq \min _{0 \neq x \in \operatorname{ker}(B) \cap F ;\|x\|=1}\langle(A+B) x, x\rangle .
$$

So, we conclude that

$$
\lambda_{n}(A+B) \geq \lambda_{n+\operatorname{rank}(B)}(A) .
$$

The second inequality in (A.6) of this lemma is obtained when we put $A^{\prime}=A+B$, $B^{\prime}=-B, n^{\prime}=n-\operatorname{rank}(B)$ and apply the conclusion (A.7) for $A^{\prime}, B^{\prime}$ and $n^{\prime}$ instead of $A, B$ and $n$. $\square$

We now call $\pi_{N}$ be the orthogonal projection on the first $N$ eigenvectors of $B$. 
By the property of an orthogonal projection, we can rewrite

$$
B=\pi_{N} B \pi_{N}+\left(I-\pi_{N}\right) B\left(I-\pi_{N}\right),
$$

then

$$
A+B=A+\pi_{N} B \pi_{N}+\left(I-\pi_{N}\right) B\left(I-\pi_{N}\right) .
$$

We note that

$$
\operatorname{rank}\left(\pi_{N} B \pi_{N}\right)=N
$$

and

$$
\left\|\left(I-\pi_{N}\right) B\left(I-\pi_{N}\right)\right\| \leq \lambda_{N}(B) .
$$

By applying lemma A.3. we get $\forall n \geq N$,

$$
\begin{aligned}
& \lambda_{n}\left(A+\pi_{N} B \pi_{N}\right)-\left\|\left(I-\pi_{N}\right) B\left(I-\pi_{N}\right)\right\| \leq \lambda_{n}(A+B), \\
& \lambda_{n}(A+B) \leq \lambda_{n}\left(A+\pi_{N} B \pi_{N}\right)+\left\|\left(I-\pi_{N}\right) B\left(I-\pi_{N}\right)\right\| .
\end{aligned}
$$

From (A.8), we obtain

$$
\lambda_{n}\left(A+\pi_{N} B \pi_{N}\right)-\lambda_{N}(B) \leq \lambda_{n}(A+B) \leq \lambda_{n}\left(A+\pi_{N} B \pi_{N}\right)+\lambda_{N}(B) .
$$

According to lemma A.4

$$
\lambda_{n+N}(A)-\lambda_{N}(B) \leq \lambda_{n}(A+B) \leq \lambda_{n-N}(A)+\lambda_{N}(B) .
$$

We can thus conclude that

$$
\forall N, \forall n \geq N: \lambda_{n+N}(A)-\lambda_{N}(B) \leq \lambda_{n}(A+B) \leq \lambda_{n-N}(A)+\lambda_{N}(B) .
$$

Lemma A.5. Let $\left\{\lambda_{n}(A)\right\}$ and $\left\{\lambda_{n}(B)\right\}$ are the eigenvalues of two self-adjoint operators $A$ and $B$. If $\lambda_{n}(A) \sim c n^{-s}$ and $\lambda_{N}(B)=\rho^{N}$ where $A$ and $\rho$ are some constants, $0 \leq \rho \leq 1$, then

$$
\lambda_{n}(A+B) \sim c n^{-s} .
$$

Proof. Indeed, by applying (A.9), if we choose $N(n)=n^{\delta}$, with $\delta<1$, then we obtain from (A.9) that

$$
c\left(n+n^{\delta}\right)^{-s}(1+o(1))-\rho^{n^{\delta}} \leq \lambda_{n}(A+B) \leq c\left(n-n^{\delta}\right)^{-s}(1+o(1))+\rho^{n^{\delta}},
$$

Let $n \rightarrow \infty$, we have

$$
c n^{-s}(1+o(1)) \leq \lambda_{n}(A+B) \leq c n^{-s}(1+o(1)) .
$$

Hence, we conclude that $\lambda_{n}(A+B) \sim c n^{-s}$.

We now turn back to prove the Proposition A.1.

We consider the eigenpairs of the operator $V \tilde{T} V$, where $\tilde{T}$ is defined in Eq. (2.7), and $V$ is defined by

$$
V(\cos n x)=\sqrt{1-(1-a)^{n}} \cos n x \simeq\left(1-\frac{1}{2}(1-a)^{n}\right) \cos n x
$$


(the last approximate equality is valid for $a \ll 1$ ). We note that $V$ and $\tilde{T}$ are selfadjoint operators. We rewrite $V$ as $I-R$ where

$$
R(\cos n x)=\frac{1}{2}(1-a)^{n} \cos n x
$$

then,

$$
V \tilde{T} V=(I-R) \tilde{T}(I-R)=\tilde{T}-R \tilde{T}-\tilde{T} R-R \tilde{T} R .
$$

Let us denote by $K_{N}$ the image of the orthonormal projection on the first $N^{\text {th }}$ eigenvectors of $R$ and $R_{N}$ the image of the orthonormal projection on the rest eigenvectors of $R$. By definition, $R=K_{N}+R_{N}$. Then,

$$
\begin{aligned}
V \tilde{T} V & =\tilde{T}-\left(K_{N}+R_{N}\right) \tilde{T}-\tilde{T}\left(K_{N}+R_{N}\right)+\left(K_{N}+R_{N}\right) \tilde{T}\left(K_{N}+R_{N}\right) \\
& =\tilde{T} \underbrace{-K_{N} \tilde{T}-\tilde{T} K_{N}+K_{N} \tilde{T} K_{N}+K_{N} \tilde{T} R_{N}+R_{N} \tilde{T} K_{N}}_{\text {these operators have the finite rank, which equal to } N}-R_{N} \tilde{T}-\tilde{T} R_{N}+R_{N} \tilde{T} R_{N} .
\end{aligned}
$$

We note that $\operatorname{rank}\left(K_{N}\right)=N$ and in formula (A.10), whenever there is a $K_{N}$, we have an operator of rank $N$. Moreover, $-R_{N} \tilde{T}-\tilde{T} R_{N}+R_{N} \tilde{T} R_{N}$ has the norm dominated by the $N^{\text {th }}$ eigenvalue of $R$ :

$$
\begin{aligned}
\left\|-R_{N} \tilde{T}-\tilde{T} R_{N}+R_{N} \tilde{T} R_{N}\right\| & \leq\left\|R_{N} \tilde{T}\right\|+\left\|\tilde{T} R_{N}\right\|+\left\|R_{N} \tilde{T} R_{N}\right\| \\
& \leq c\left\|R_{N}\right\| \leq c \lambda_{N}(R)=\frac{c}{2}(1-a)^{N} .
\end{aligned}
$$

Since the operator $\tilde{T}$ is the solution of the Sturm-Liouville problem, $\lambda_{n}(\tilde{T}) \sim A_{\epsilon} n^{-2}$. Hence, refer to lemma A.5. we get that

$$
\lambda_{n}(V \tilde{T} V) \sim A_{\epsilon} n^{-2} .
$$

0

B. Numerical computation of spectral characteristics. We briefly present a numerical algorithm to compute the spectral characteristics $\lambda_{n}$ and $\psi_{n}$. In order to compute the eigenvalues $\lambda_{n}$ and the eigenvectors $e_{n}$ of the operator $V \tilde{T} V$, we get an explicit representation of this operator in the basis $\cos n \theta$. First, we find

$$
\tilde{T}(\cos n \theta)= \begin{cases}\frac{\cos n \theta-\cos n(\pi-\epsilon)}{n^{2}}, & 0 \leq \theta<\pi-\epsilon, \\ 0, & \pi-\epsilon \leq \theta \leq \pi,\end{cases}
$$

and

$$
\tilde{T}(1)(\theta)= \begin{cases}\frac{(\pi-\epsilon)^{2}-\theta^{2}}{2}, & 0 \leq \theta<\pi-\epsilon, \\ 0, & \pi-\epsilon \leq \theta \leq \pi,\end{cases}
$$

from which the expansion of $\tilde{T}(\cos n \theta)(n \geq 0)$ in the basis $\{\cos n \theta\}$ of $L_{\text {even }}^{2}[0, \pi]$ is

$$
\tilde{T}(\cos n \theta)=\sum_{m \geq 0} \mathbf{T}_{m n} \cos m \theta \quad n \geq 0,
$$


where the coefficients $\mathbf{T}_{m n}$ are defined by

$$
\begin{aligned}
\frac{\pi}{2} \mathbf{T}_{m n}= & \begin{cases}\langle\tilde{T}(\cos n \theta), \cos m \theta\rangle, & \text { if } m \geq 1 \\
\frac{1}{2}\langle\tilde{T}(\cos n \theta), 1\rangle, & \text { if } m=0\end{cases} \\
= & \begin{array}{ll}
\frac{1}{2}\left(1-\delta_{m n}\right) \frac{(-1)^{m+n+1}}{m n}\left[\frac{\sin (m-n) \epsilon}{m-n}-\frac{\sin (m+n) \epsilon}{m+n}\right]+\frac{1}{2} \delta_{m n} \frac{1}{n^{2}}\left(\pi-\epsilon+\frac{\sin 2 n \epsilon}{2 n}\right) \\
\frac{1}{2} \frac{(-1)^{n+1}}{n^{2}}\left[(\pi-\epsilon) \cos n \epsilon+\frac{\sin n \epsilon}{n}\right], & (m \geq 1 ; n \geq 1), \\
\frac{(-1)^{m+1}}{m^{2}}\left[(\pi-\epsilon) \cos m \epsilon+\frac{\sin m \epsilon}{m}\right], & (m \geq 1 ; n=0), \\
\frac{(\pi-\epsilon)^{3}}{6}, & (m=0 ; n=0) .
\end{array}
\end{aligned}
$$

In turn, the operator $V$ has a diagonal representation:

$$
\mathbf{V}_{m n}= \begin{cases}\sqrt{1-(1-a)^{m}} & (m=n ; m, n \geq 0), \\ 0 & (m \neq n ; m, n \geq 0) .\end{cases}
$$

Combining these results, the operator $V \tilde{T} V$ is represented by the infinite-dimensional matrix VTV whose elements are

$$
\begin{aligned}
{[\mathbf{V T V}]_{m, n} } & =\frac{1}{\pi} \sqrt{1-(1-a)^{n}} \sqrt{1-(1-a)^{m}}\left\{\delta_{m n} \frac{1}{n^{2}}\left(\pi-\epsilon+\frac{\sin 2 n \epsilon}{2 n}\right)\right. \\
& \left.-\left(1-\delta_{m n}\right) \frac{(-1)^{m+n}}{m n}\left[\frac{\sin (m-n) \epsilon}{m-n}-\frac{\sin (m+n) \epsilon}{m+n}\right]\right\} \quad(m \geq 1 ; n \geq 1),
\end{aligned}
$$

and $[\mathbf{V T V}]_{m, n}=0$ if $m=0$ or $n=0$. Solving the eigenvalues $\left\{\lambda_{n}\right\}$ and the eigenvectors $\left\{e_{n}\right\}$ of the operator $V \tilde{T} V$ is equivalent to finding the eigenpairs of the associated matrix VTV. Note that this matrix is symmetric.

The matrix VTV is diagonalized in Matlab that finds the eigenvalues $\lambda_{n}$ and the coefficients $v_{m n}$ determining the orthonormal basis $\left\{e_{n}\right\}_{n \geq 0}$ as

$$
e_{n}(\theta)=\sqrt{\frac{1}{\pi}} v_{0 n}+\sqrt{\frac{2}{\pi}} \sum_{m \geq 1} v_{m n} \cos m \theta .
$$

The spectral weights $\psi_{n}$ are then given as

$$
\psi_{n}=\left\langle\psi, e_{n}\right\rangle=\sqrt{\frac{2}{\pi}} \sum_{m \geq 1} v_{m n}\langle\psi, \cos m \theta\rangle,
$$

where

$$
\langle\psi, \cos m \theta\rangle=\sqrt{1-(1-a)^{m}} \frac{(-1)^{m+1}}{m^{2}}\left[(\pi-\epsilon) \cos m \epsilon+\frac{\sin m \epsilon}{m}\right],
$$

and $\langle\psi, 1\rangle=\langle V \tilde{T}(1), 1\rangle=\langle\tilde{T}(1), V 1\rangle=0$.

C. Pure bulk diffusion phase. In [15, the mean exit time for pure bulk diffusion phase $(\lambda=\infty, a=0)$ was found to be

$$
t_{2}(r, \theta)=\frac{1}{D_{2}}\left(\frac{1-r^{2}}{4}+\frac{\alpha_{0}}{2}+\sum_{n=1}^{\infty} \alpha_{n} r^{n} \cos n \theta\right),
$$


FIG. C.1. Mean exit times $\left\langle t_{1}\right\rangle_{\lambda=0}$ and $\left\langle t_{1}\right\rangle_{\lambda=\infty}$ from Eqs. 2.29 and C.5 for surface diffusion and pure bulk diffusion, as a function of $\epsilon$. These times are multiplied by the corresponding diffusion coefficients $D_{1}$ and $D_{2}$.

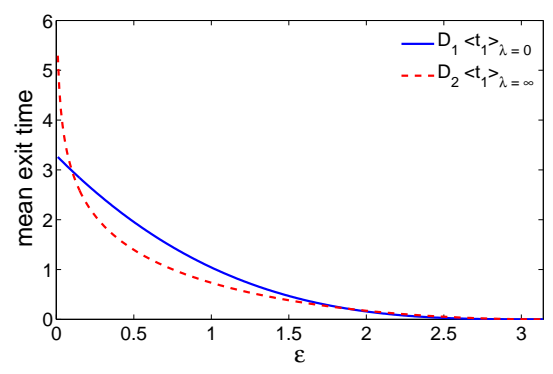

where

$$
\begin{aligned}
\alpha_{0} & =-2 \ln [\sin (\epsilon / 2)], \\
\alpha_{n} & =\frac{(-1)^{n-1}}{2 n}\left[P_{n}(\cos \epsilon)+P_{n-1}(\cos \epsilon)\right],
\end{aligned}
$$

where $P_{n}(z)$ are Legendre polynomials. In the limit $r \rightarrow 1$, one gets

$$
t_{1}(\theta)= \begin{cases}\frac{1}{D_{2}}\left(\frac{1}{2} \alpha_{0}+\sum_{n=1}^{\infty} \alpha_{n} \cos n \theta\right) & (0<\theta<\pi-\epsilon), \\ 0, & (\pi-\epsilon \leq \theta \leq \pi) .\end{cases}
$$

The average of the uniformly distributed starting point $\theta$ yields

$$
\left\langle t_{1}\right\rangle_{b}=\frac{1}{\pi D_{2}}\left(-(\pi-\epsilon) \ln [\sin (\epsilon / 2)]+\sum_{n=1}^{\infty} \frac{\sin n \epsilon}{2 n^{2}}\left[P_{n}(\cos \epsilon)+P_{n-1}(\cos \epsilon)\right]\right)
$$

For small $\epsilon$, the first term dominates yielding $\left\langle t_{1}\right\rangle_{b} \simeq \frac{\ln (2 / \epsilon)}{D_{2}}(1+O(\epsilon))$.

Figure C.1 shows the mean exit times $\left\langle t_{1}\right\rangle_{\lambda=0}$ and $\left\langle t_{1}\right\rangle_{\lambda=\infty}$ from Eqs. (2.29) and (C.5) for surface diffusion and pure bulk diffusion, as a function of $\epsilon$.

D. Transportation case $(a=1)$. As we earlier discussed, one typically considers small values of the ejection distance $a$. Nevertheless, the above results are applicable to any value of $a$ from 0 to 1 . The so-called transportation case $a=1$ when the particle is reflected to the origin, was studied by Bénichou et al. 33. In this case, successive explorations between any two reflections are independent that allows one to get much simpler formulas. For instance, in the limit $\lambda \rightarrow \infty$, the Laplace transformed probability density of the exit time, $\mathcal{L}[P(t)](s)$ (with a uniformly chosen initial point on the circle), has a simple expression: $\mathcal{L}[P(t)](s)=q\left[1-(1-q) / I_{0}\left(\sqrt{s / D_{2}}\right)\right]^{-1}$, where $q=\epsilon / \pi$, and $1 / I_{0}\left(\sqrt{s / D_{2}}\right)$ is the Laplace transformed probability density for the first passage time to the circle when started from the origin (with $I_{0}(z)$ being the modified Bessel function of the first kind). As a consequence, the mean exit is simply

$$
\mathcal{T}_{a=1}=\frac{\pi-\epsilon}{4 D_{2} \epsilon}
$$

This limit is clearly seen on Fig. 3.5 


\section{REFERENCES}

[1] O. Bénichou, D. S. Grebenkov, P. E. Levitz, C. Loverdo, and R. Voituriez. Optimal reaction time for surface-mediated diffusion. Phys. Rev. Lett., 105, 2010.

[2] O. Bénichou, D. S. Grebenkov, P. E. Levitz, C. Loverdo, and R. Voituriez. Mean first-passage time of surface-mediated diffusion in spherical domains. J. Stat. Phys., 142(4):657-685, 2011.

[3] O. Bénichou, C. Loverdo, M. Moreau, and R. Voituriez. Intermittent search strategies. Rev. Mod. Phys., 83, 2011.

[4] P. C. Bressloff and J. M. Newby. Stochastic models of intracellular transport. Rev. Mod. Phys., 85, 2013.

[5] T. Calandre, O. Bénichou, D. S. Grebenkov, and R. Voituriez. The interfacial territory covered by surface-mediated diffusion. Phys. Rev. E, 85, 2012.

[6] T. Calandre, O. Bénichou, D. S. Grebenkov, and R. Voituriez. Splitting probabilities and interfacial territory covered by $2 \mathrm{~d}$ and $3 \mathrm{~d}$ surface-mediated diffusion. Phys. Rev. E, 89, 2014.

[7] S. A. Isaacson and J. M. Newby. Uniform asymptotic approximation of diffusion to a small target. Phys. Rev. E, 88, 2013.

[8] R. Metzler, G. Oshanin, and S. Redner, editors. First-Passage Phenomena and Their Applications. World Scientific Publisher, 2014.

[9] S. Redner. A Guide to First-Passage Processes. Cambridge University Press, Cambridge, 2001.

[10] J. Reingruber and D. Holcman. Diffusion in narrow domains and application to phototransduction. Phys. Rev. E, 79, 2009.

[11] J.A. Revelli, C.E. Budde, O. Prato, D. Deza, and H.S. Wio. Bulk mediated surface diffusion: non markovian desorption with finite first moment. Eur. Phys. J. B, 43:65, 2005.

[12] F. Rojo and C.E Budde. Enhanced diffusion through surface excursion: A master-equation approach to the narrow-escape-time problem. Phys. Rev. E, 84:021117, 2011.

[13] F. Rojo, C.E Jr. Budde, H.S. Wio, and C.E Budde. Enhanced transport through desorptionmediated diffusion. Phys. Rev. E, 87:012115, 2013.

[14] F. Rojo, P.A. Pury, and C.E Budde. Intermittent pathways towards a dynamical target.

[15] J.-F. Rupprecht, O. Bénichou, D. S. Grebenkov, and R. Voituriez. Exit time distribution in spherically symmetric two-dimensional domains. J. Stat. Phys.

[16] J.-F. Rupprecht, O. Bénichou, D. S. Grebenkov, and R. Voituriez. Exact mean exit time for surface-mediated diffusion. Phys. Rev. E, 86, 2012.

[17] J.-F. Rupprecht, O. Bénichou, D. S. Grebenkov, and R. Voituriez. Kinetics of active surfacemediated diffusion in spherically symmetric domains. J. Stat. Phys., 147, 2012.

[18] Z. Schuss, A. Singer, and D. Holcman. The narrow escape problem for diffusion in cellular microdomains. Proc. Nat. Ac. Sci., 104:16098, 2007.

[19] A. Singer, Z. Schuss, and D. Holcman. Narrow escape. II. The circular disk. J. Stat. Phys., 122(3):465-489, 2006.

[20] A. Singer, Z. Schuss, and D. Holcman. Narrow escape. III. Non-smooth domains and Riemann surfaces. J. Stat. Phys., 122(3):491-509, 2006.

[21] A. Singer, Z. Schuss, D. Holcman, and R. S. Eisenberg. Narrow escape. I. J. Stat. Phys., 122(3):437-463, 2006. 